Published in final edited form as:

Anal Chem. 2007 April 1; 79(7): 2756-2761.

\title{
Using a Multijunction Microfluidic Device To Inject Substrate into an Array of Preformed Plugs without Cross-Contamination:
}

\author{
Comparing Theory and Experiments
}

\author{
Liang Li, James Q. Boedicker, and Rustem F. Ismagilov* \\ Department of Chemistry and Institute for Biophysical Dynamics, The University of Chicago, 929 \\ East 57th Street, Chicago, Illinois 60637
}

\section{Abstract}

\begin{abstract}
In this paper we describe a multijunction microfluidic device for the injection of a substrate into an array of preformed plugs carried by an immiscible fluid in a microchannel. The device uses multiple junctions to inject substrate into preformed plugs without synchronization of the flow of substrate and the array of preformed plugs of reagent, which reduces cross-contamination of the plugs, eliminates formation of small droplets of substrate, and allows a greater range of injection ratios compared to that of a single T-junction. The device was based on a previously developed physical model for transport that was here adapted to describe injection and experimentally verified. After characterization, the device was applied to two biochemical assays, including evaluation of the enzymatic activity of thrombin and determination of the coagulation time of human blood plasma, which both provided reliable results. The reduction of cross-contamination and greater range of injection ratios achieved by this device may improve the processes that involve addition and titration of reagents into plugs, such as high-throughput screening of protein crystallization conditions.
\end{abstract}

In this paper we discuss a physical model of multiphase fluid flow ${ }^{1,2}$ during injection of a stream into droplets and the use of this model to design and validate a multijunction microfluidic injector for reliable addition of a substrate into an array of preformed plugs containing reagents. This model has been presented previously for multiphase separation, 1,2 and we used it to describe the related process of injecting reagents into droplets. Microfluidic systems are attractive for miniaturizing laboratory techniques, ${ }^{3-8}$ and systems with multiphase flows are useful for compartmentalizing reagents, enhancing mixing, and reducing dispersion but require improved understanding and control. ${ }^{2,3,6,9-13}$ In plug-based systems, nanoliter or picoliter droplets are formed within microchannels and carried by an immiscible fluid. ${ }^{14-16}$ Each plug contains multiple reagents and can act as a microreactor. ${ }^{13,17-21}$ For chemical and biological reactions and analysis, multiple substrates and reagents must be introduced into plugs. Introducing multiple reagents as a plug is forming can be done simply by relying on laminar flow of several streams containing reagents. ${ }^{13}$ However, reliable addition of a substrate to preformed plugs is more challenging.

Injection into preformed plugs may improve a number of processes such as protein crystallization, ${ }^{22}$ synthesis of particles, ${ }^{23}$ biological assays, ${ }^{22}$ combinatorial chemistry, ${ }^{24}$ and chemical synthesis with one or multiple steps. ${ }^{25,26}$ In a T-junction, substrate is injected from the side channel into preformed plugs traveling in the main channel (Figure 1A). Three problems were identified for injection using a T-junction: (i) Cross-contamination between plugs occurred when the substrate stream picked up reagents from a preformed plug and

\footnotetext{
*To whom correspondence should be addressed. E-mail: r-ismagilov@uchicago.edu. SUPPORTING INFORMATION AVAILABLE 
injected them into the subsequent plug in the array, a problem further described in the Results and Discussion. 22,24 "Blank plugs", or buffer plugs, could be inserted between preformed plugs to remove the contaminant from the substrate stream before the subsequent plug arrived, thereby reducing cross-contamination but wasting substrate. (ii) Limited volumes of substrate could be injected into each plug. ${ }^{24,27}$ (iii) At higher injection ratios, defined as the volume of substrate injected divided by the original volume of the plug, substrate droplets formed between preformed plugs.

To overcome the limitations present in the T-junction, we designed a multijunction injection device (Figure 1B) and defined operation parameters for the device based a physical model of multiphase fluid flow, 1,2 which we adapted to describe the injector and experimentally verified. To address problems $\mathrm{i}$ and ii, the device was designed with multiple long, narrow hydrophilic side channels, which prevented cross-contamination while increasing the volume of substrate that could be injected into each plug. To address problem iii, we used a physical model $^{1,2}$ to define the maximum working flow rate, $Q_{\max }$, of the device. At a flow rate of substrate below $Q_{\max }$, substrate was reliably injected into preformed plugs without the formation of substrate droplets. This device continuously injected substrate through any of the side channels that were in contact with a preformed plug at a given time, eliminating the need to synchronize the arrival of a plug at the side channels with the flow of substrate.

\section{EXPERIMENTAL SECTION}

\section{Reagents and Solutions}

Perfluoroperhydrophenanthrene (PPP) and $1 H, 1 H, 2 H, 2 H$-perfluorooctanol (PFO) were obtained from Alfa Aesar (Ward Hill, MA). Substrate fluorescein diphosphate (FDP) and thrombin from human plasma (1370 units/mg of protein) were obtained from Sigma-Aldrich (St. Louis, MO). Substrate Boc-Asp (oBzl)-Pro-Arg-MCA (MCA IIa) and substrate Boc-IleGln-Gly-Arg-MCA (MCA Xa) were obtained from the Peptide Institute, Inc. (Osaka, Japan). Alexin was obtained from Trinity Biotech Plc, Co. (Wicklow, Ireland). Human pooled plasma (pooled normal) was obtained from George King Bio-medical, Inc. (Overland Park, KS). Red dye solution (referred to as "red dye") was made by mixing McCormick red food dye 1:100 (v/v) with Millipore water. Green dye solution (referred to as "green dye") and viscous green dye solution (referred to as "viscous green dye") were made by mixing McCormick green food dye $1: 10(\mathrm{v} / \mathrm{v})$ with $24 \%(\mathrm{w} / \mathrm{w})$ and $68 \%(\mathrm{w} / \mathrm{w})$ glycerol/0.2 $\mathrm{M} \mathrm{KNO}_{3}$ solution, respectively.

\section{Fabrication of the Multijunction Device}

Microfluidic devices were fabricated using rapid prototyping in poly(dimethylsiloxane) (PDMS) from masters fabricated by using two-layer lithography as previously described. ${ }^{27}$ Microchannels were rendered hydrophobic and fluorophilic by using the silanization protocol described previously. ${ }^{28}$ The parallel side channels were cut off and replaced with glass capillaries (i.d. $100 \mu \mathrm{m}$, rendered hydrophilic by using a Plasma Prep II plasma cleaner). Capillary wax was used to seal the connections to prevent leaking. A schematic drawing of this device is shown in Figure 2.

\section{Validation of the Physical Model}

The experimental setup is shown in Figure 3.

Parameters and Solutions-PFO/PPP $(1: 10, \mathrm{v} / \mathrm{v})$ was used as the carrier fluid. The dynamic viscosity of the green dye was $0.0019 \mathrm{~Pa} \mathrm{~s}$, and the surface tension between the carrier fluid and the green dye was $0.0079 \mathrm{~N} / \mathrm{m}$. Flow rates of carrier fluid were tested in the range of $0.5-4.0 \mu \mathrm{L} / \mathrm{min}$. Flow rates of the red dye and the green dye were kept the same as that of the carrier fluid. For flow rates less than $2.0 \mu \mathrm{L} / \mathrm{min}$, the array of red plugs was first formed at 4.0 
$\mu \mathrm{L} / \mathrm{min}$ for the red dye and $4.0 \mu \mathrm{L} / \mathrm{min}$ for the carrier fluid. The array was driven by carrier fluid at a flow rate of 1.0-4.0 $\mu \mathrm{L} / \mathrm{min}$, twice the testing flow rate $(0.5-2.0 \mu \mathrm{L} / \mathrm{min})$. The viscosity was measured with a viscometer (Cannon, Inc., State College, PA), and the surface tension was measured with the reported hanging drop method. ${ }^{28}$

Testing the Effect of Surface Tension ( $y)$ at the Substrate/Carrier Interface on $\mathbf{Q}_{\max }$ - The PFO concentration in the carrier fluid was reduced to 1:500 (v/v), and the surface tension between the green dye and the carrier fluid was $0.0202 \mathrm{~N} / \mathrm{m}$. Flow rates of carrier fluid were tested in the range of 2.0-8.0 $\mu \mathrm{L} / \mathrm{min}$. Flow rates of the red and green dyes were kept the same as that of the carrier fluid.

Testing the Effect of the Viscosity $(\boldsymbol{\mu})$ of the Substrate on $\mathbf{Q}_{\max }$-The dynamic viscosity of the viscous green dye was $0.013 \mathrm{~Pa}$ s. The surface tension between the carrier fluid and the viscous green dye was $0.0076 \mathrm{~N} / \mathrm{m}$. Flow rates of the carrier fluid were tested in the range of $0.1-0.4 \mu \mathrm{L} / \mathrm{min}$, and flow rates of the red and green dyes were kept the same as that of the carrier fluid. The array of red plugs was formed as described above and driven by carrier fluid at a flow rate of $0.2-0.8 \mu \mathrm{L} / \mathrm{min}$, twice the testing flow rate $(0.1-0.4 \mu \mathrm{L} / \mathrm{min})$.

\section{Test of Cross-Contamination}

Color Change Reaction-Simple T-junction and multijunction injectors were used to inject $0.6 \mathrm{M} \mathrm{KSCN}$ solution into preformed plugs of $0.2 \mathrm{M} \mathrm{Fe}\left(\mathrm{NO}_{3}\right)_{3}$ solution (Figure 2). The carrier fluid was PFO/PPP $(1: 10, \mathrm{v} / \mathrm{v})$. The flow rate of the array of preformed plugs was $2.0 \mu \mathrm{L} / \mathrm{min}$ (1.0 $\mu \mathrm{L} / \mathrm{min}$ for both the carrier fluid and $\mathrm{Fe}\left(\mathrm{NO}_{3}\right)_{3}$ solution), and that of the KSCN solution was $1.0 \mu \mathrm{L} / \mathrm{min}$.

Fluorescence Test-An array of alternating plugs with and without fluorescein was generated by aspirating $\sim 27 \mathrm{~nL}$ of reagents in alternation with air bubbles $\sim 9 \mathrm{~nL}$ in volume. The fluorescein solution was $0.10 \mathrm{mM}$ fluorescein in $1 \times \mathrm{PBS}$ buffer, and the non-fluorescein solution was $1 \times \mathrm{PBS}$ buffer. The carrier fluid was PFO/PPP $(1: 10, \mathrm{v} / \mathrm{v})$. This array was aspirated into a piece of Teflon tubing (o.d. $250 \mu \mathrm{m}$, i.d. $200 \mu \mathrm{m}$, length $\sim 8 \mathrm{~cm}$ ). Capillary wax was used to seal one end of the Teflon tubing containing the array to 30 gauge Teflon tubing connected to a $10 \mu \mathrm{L}$ syringe with a removable 27 gauge needle. The syringe and 30 gauge Teflon tubing were filled with PFO/PPP $(1: 10, \mathrm{v} / \mathrm{v})$.

The other end of the Teflon tubing containing the array was sealed to the inlet of the multijunction device with capillary wax, and the array of plugs was injected with nonfluorescent $1 \times$ PBS buffer (Supporting Information Figure S-1). The flow rate of the array was $1.0 \mu \mathrm{L} / \mathrm{min}$, and the flow rate of the buffer was $0.5 \mu \mathrm{L} / \mathrm{min}$. The fluorescence intensity of the resulting plugs was measured with a fluorescence microscopy systems a Leica DMI6000 microscope (Leica Microsystem, Germany) with a 10×/0.4 NA Leica objective and a Hamamatsu ORCA-ER camera. A GFP filter was used to obtain fluorescent images of the resulting plugs. The images were analyzed with the Metamorph imaging system, version 6.3r1. Cross-contamination was quantified by determining the ratio of the intensity of the buffer plug to the intensity of the fluorescein plug. The ratio was $1.28 \times 10^{-3}$.

To detect any cross-contamination from manual aspiration, another array of plugs was made in the same configuration. Without injection, the plugs were directly analyzed with fluorescence microscopy in the same manner as above. The cross-contamination from manual aspiration was $0.4 \times 10^{-4}$. We concluded that the cross-contamination from injection was (1.28 $\left.\times 10^{-3}\right)-\left(0.4 \times 10^{-4}\right)=1.2 \times 10^{-3}$. 


\section{Test of the Injection Ratio and Consistency}

Plugs of red dye were formed in PFO/PPP (1:500, v/v) at flow rate of $10.0 \mu \mathrm{L} / \mathrm{min}$ for both the red dye and the carrier fluid. The array of plugs was then driven by the carrier fluid at $4.0 \mu \mathrm{L} /$ min. We assumed the plugs were formed at $Q_{\text {red }}=4 \times 10 /(10+10)=2.0 \mu \mathrm{L} / \mathrm{min}$. The green dye was injected using the multijunction device. The flow rate of the green dye was changed from 0 to $5.0 \mu \mathrm{L} / \mathrm{min}$. For each flow rate, the plug length was measured.

\section{Control Assay To Test the Enzymatic Activity of Three Substrates}

An array of plugs of three substrates (150 $\mu \mathrm{M}$ substrate MCA IIa in $20 \mathrm{mM}$ Tris, pH 7.3, 150 $\mu \mathrm{M}$ substrate MCA Xa in $20 \mathrm{mM}$ Tris, $\mathrm{pH} 7.3$, and $11 \mu \mathrm{M}$ FDP in $1 \times \mathrm{PBS}$ ) was prepared in a piece of Teflon tubing as in the fluorescence test. The array of plugs with a flow rate of 1.0 $\mu \mathrm{L} / \mathrm{min}$ was injected with thrombin $(0.8 \mu \mathrm{M}$ in $20 \mathrm{mM}$ Tris, $\mathrm{pH} 7.3)$ at $0.5 \mu \mathrm{L} / \mathrm{min}$. The resulting plugs were collected in a piece of Teflon tubing for fluorescence detection (Supporting Information Figure S-2). The same fluorescent microscopy system used for the fluorescence test was employed here, and GFP and DAPI filters were used to take images. The images were analyzed with Metamorph.

\section{Control Assay To Test Blood Clotting}

An array of five sample plugs was prepared in a piece of Teflon tubing as in the fluorescence test in the following sequence: (1) Tris buffer (20 mM Tris, $0.1 \mathrm{M} \mathrm{NaCl}, \mathrm{pH} 7.4)$, (2) human pooled plasma (PP), (3) thrombin (0.8 $\mu \mathrm{M}$ in $18 \mathrm{mM}$ Tris and $0.09 \mathrm{M} \mathrm{NaCl}, \mathrm{pH}$ 7.4), (4) $\mathrm{PP}+$ alexin $(1: 1, \mathrm{v} / \mathrm{v}),(5)$ Tris buffer. The array was injected with the substrate containing $150 \mu \mathrm{M}$ MCA IIa, $40 \mathrm{mM} \mathrm{CaCl}_{2}, 20 \mathrm{mM}$ Tris, and $0.1 \mathrm{M} \mathrm{NaCl}, \mathrm{pH} 7.4$, using the multijunction device (Figure 5A,B). The flow rate of the array was $0.5 \mu \mathrm{L} / \mathrm{min}$, and the flow rate of the mixture was $0.25 \mu \mathrm{L} / \mathrm{min}$. The resulting plugs were collected in a piece of Teflon tubing and monitored with fluorescence microscopy for 60 min using a DAPI filter.

\section{RESULTS AND DISCUSSION}

\section{Reducing Cross-Contamination}

To reduce cross-contamination, we designed a multijunction device with three long, narrow hydrophilic side channels. We previously found that creating a hydrophilic side channel made injection more reliable, especially when the side channel was narrow. ${ }^{27}$ This modification was achieved by replacing the PDMS side channel with a thin glass capillary. As narrow side channels have also been found to reduce cross-contamination, ${ }^{27}$ we designed side channels to be narrow and long by using a two-layer geometry (Figure 2). This decrease of crosscontamination can be explained by an increase of the Peclet number $(\mathrm{Pe}) . \mathrm{Pe}$ is a dimensionless parameter that represents the ratio of the rate of convection to the rate of diffusion. During injection, convection injects the substrate into the plug, and diffusion of reagents out of the plug causes contamination of the substrate stream (Figure 4A). Reducing the size of the side channels increases the velocity $U(\mathrm{~m} / \mathrm{s})$ of the substrate given the same volumetric flow rate. Since $P e$ is defined as $U d / D$, where $U$ is the flow velocity of the substrate $(\mathrm{m} / \mathrm{s}), d(\mathrm{~m})$ is a characteristic dimension, and $D\left(\mathrm{~m}^{2} / \mathrm{s}\right)$ is the diffusion coefficient, increasing $U$ results in an increase of $P e$, less effective diffusion, and reduced cross-contamination.

However, using narrow side channels limits the amount of substrate that could be injected into the plugs. In a T-junction, the injection ratio was below $0.5 .{ }^{27}$ At higher injection ratios, the substrate formed separate droplets between preformed plugs instead of being injected into a plug. Small substrate droplets eventually coalesced with one of their neighboring plugs, making the volume increase in the plugs inconsistent. This limitation made some applications difficult. For example, protein crystallization, in principle, needs an injection ratio of 1, corresponding 
to equal volumes of precipitant and protein solution in a mixture. Coalescence of small substrate droplets also made the time of reaction initiation difficult to define, limiting the applicability of this technique to kinetic studies.

\section{Eliminating the Formation of Substrate Droplets}

To achieve a broader range of injection ratios while eliminating the formation of substrate droplets, we wished to find conditions under which capillary forces would prevent injection of the substrate into the carrier fluid through channels not in contact with a preformed plug (and prevent formation of small substrate droplets). Using previously described equations, 1 , 2,29 we described the flow of the substrate into the main channel by considering the competition between the capillary pressure $\left(P_{\text {cap }}\right)$ and pressure drop $\left(\Delta P_{\text {flow }}\right)$, expressed as eqs 1 and 2 ,

$$
\begin{gathered}
P_{\text {cap }}=(2 \gamma \cos \theta) / R \\
\Delta P_{\text {flow }}=(8 Q \mu L) /\left(\pi R^{4}\right)
\end{gathered}
$$

respectively, where $P_{\text {cap }}(\mathrm{Pa})$ is the capillary pressure exerted on the substrate by the carrier fluid, $\gamma(\mathrm{N} / \mathrm{m})$ is the surface tension between the substrate and the carrier fluid, $R(\mathrm{~m})$ is the radius of the circular side channels, $\theta$ (degree) is the angle between the substrate and the carrier fluid, $Q\left(\mathrm{~m}^{3} / \mathrm{s}\right)$ is the volumetric flow rate of the substrate, $\mu(\mathrm{Pa} \mathrm{s})$ is the dynamic viscosity of the substrate, $L(\mathrm{~m})$ is the length of the side channel, and $\Delta P_{\text {flow }}$ is the pressure drop along the side channels with length $L(\mathrm{~m})$ (Figure 1B). When a preformed plug is in contact with the substrate (Figure 1B, right inset), $P_{\text {cap }}$ exerted on the substrate is reduced and injection proceeds. When no plug is present at the junction, capillary pressure prevents injection of substrate into the carrier fluid (Figure 1B, left inset), which can be expressed by

$$
P_{\text {cap }}>\Delta P_{\text {flow }}
$$

We have assumed that $P_{\text {cap }}$ is small at junctions where a plug is present and can be neglected; if this is not the case, $\Delta P_{\text {cap }}$ should be used in eq 3 . Equation 3 also requires that a preformed plug is always in contact with at least one side channel, which can only be achieved when the distance between two adjacent plugs is smaller than the distance between the two outer side channels. Equation 3 assumes that $\Delta P_{\text {flow }}$ is much higher than the pressure drop along a fluid path connecting the substrate inlet to the entrances of the side channels. Equation 3 also assumes that the system operates at a low value of the capillary number ${ }^{16,30}$ and that injection is not limited by the drainage of the carrier fluid between the substrate and the preformed plug.

Equation 3 predicts a maximum flow rate of substrate at which $\Delta P_{\text {flow }}$ can still be overcome by $P_{\text {cap }}$ and formation of substrate droplets is prevented, $Q_{\max }\left(\mathrm{m}^{3} / \mathrm{s}\right)$. Similar to the largest working flow rate of the multiphase separation device, ${ }^{1}$ this maximum flow is given by

$$
Q_{\max }=\left(\pi R^{3} \gamma\right) /(4 \mu L)
$$

when $\cos \theta=1$. We tested the influence of the $\mu$ and $\gamma$ parameters on $Q_{\max }$ in a device with $R=50 \mu \mathrm{m}$ and $L=14000 \mu \mathrm{m}$ (Figure 3 ). We used eq 4 to predict $Q_{\max }$. No adjustable parameters were used to make the predictions. Experimental values were obtained by "bracketing": changing $Q$ to identify the highest $Q$ with reliable injection and the lowest $Q$ that resulted in undesirable formation of small droplets. The predicted value of $Q_{\max }$ always fell within the "bracketed" range, confirming eq 4 . Also, both predicted and experimental values behaved as expected: $Q_{\max }$ increased as $\gamma$ was increased, and $Q_{\max }$ decreased as $\mu$ was increased (Figure $3)$. 
Therefore, for given device dimensions, substrate, and carrier fluid, a certain maximum substrate flow rate, $Q_{\max }$, exists, below which reliable injection occurs without the formation of small substrate droplets. To operate this device, an array of preformed plugs, with the distance between adjacent plugs no greater than the distance between the two outer side channels, is flowed into the device. Substrate is continuously injected at $Q<Q_{\max }$ through any of the side channels that are in contact with a preformed plug at a given time. There is no need to synchronize the arrival of a plug at the side channels with the flow of substrate, making injection reliable during continuous operation of the device.

\section{Characterizing Cross-Contamination}

We tested this device for cross-contamination, injection consistency, and the range of injection ratios. A simple T-junction and the multijunction device were both tested with a color-change reaction. Reduced contamination and more reliable injection were observed in the multijunction device compared to the T-junction (Figure 4). Cross-contamination in the multijunction device was further quantified by a fluorescence experiment. Alternating plugs with and without fluorescein, a fluorescent dye, were aspirated into a piece of Teflon tubing to form an array. This array was injected with nonfluorescent buffer using the multijunction device. Any cross-contamination from the fluorescein plug would have been injected into the next buffer plug, making the buffer plug fluoresce. Therefore, the ratio of the fluorescence intensity of the injected buffer plug to that of the injected fluorescein plug quantifies the total cross-contamination through the three side channels (Supporting Information Figure S-1). Using quantitative microscopy, we determined cross-contamination to be about $0.1 \%$ for the multijunction device, much less than that of the T-junction (up to $\sim 10 \%$ ). ${ }^{24}$

\section{Characterizating Injection Consistency and Injection Ratios}

In tests to evaluate the injection consistency and the range of injection ratios, an array of red dye plugs was generated and subsequently injected with green dye (Figure 3A). The multijunction device injected substrate over a 0-1.5 range of injection ratios (Supporting Information Figure S-3), an increase in range as compared to that of the T-junction (typically up to $\sim 0.3){ }^{27}$ The injection ratio $(v)$ was defined as the relative amount of green dye injected into the red plugs and was semiquantitatively obtained by measuring the length of the plugs after injection. The injection ratio was calculated as $v=\left(L-L_{0}\right) / L_{0}$, where $L$ is the length of the plugs after injection at a certain flow rate and $L_{0}$ is the original length of the plug. The plug length with no substrate flow (injection flow rate of $0 \mu \mathrm{L} / \mathrm{min}$ ) was used as the original length. On the basis of mass conservation, the theoretical injection ratio $v_{\text {theo }}$ is specified as $v_{\text {theo }}=$ $Q_{\text {green }} / Q_{\text {red }}$, where $Q_{\text {green }}$ is the flow rate of the green dye and $Q_{\text {red }}$ is the flow rate of the red dye $(2.0 \mu \mathrm{L} / \mathrm{min})$. We found that the injection ratio could be controlled by flow rates and could go up to 1.5 (Supporting Information Figure S-3). We also found that the amount of substrate injected into each preformed plug was consistent. The coefficient of variation $(\mathrm{CV})$ was below $3 \%$ on the basis of the measurements of six consecutive plugs.

\section{Demonstrating Potential Applications of the Multijunction Device}

We performed two control assays to demonstrate potential applications of the injection device. To ensure reliable injection, we chose flow rates of the substrate stream well below the estimated $Q_{\max }$. To check the reliability of the device for performing enzymatic activity assays, we evaluated the activity of thrombin by using three fluorogenic substrates: MCA IIa, MCA $\mathrm{Xa}$, and a negative control, FDP. Thrombin (IIa) is a protease formed during the clotting of blood that catalyzes the cleavage of peptides. When thrombin induces cleavage of a peptide from MCA IIa or MCA Xa, cumarin dye is released, resulting in an increase of fluorescence. 31 Thrombin cleaves MCA IIa 1000 times more rapidly than MCA Xa. ${ }^{31}$ An array of plugs $^{22}$ of the three substrates was injected with thrombin by using the multijunction device. 
The resulting plugs were collected in a piece of Teflon tubing for fluorescence detection (Supporting Information Figure S-2). A significant fluorescence increase was only detected in the plug of MCA IIa, indicating that the device was reliable and did not give false positive results.

To assess the capability of the device for handling complex fluids, we used the device to test the clotting time of human blood plasma. Production of thrombin is accelerated in the presence of clotting activators such as alexin. We injected the substrate MCA IIa into an array of five plugs in the following sequence: (1) Tris buffer, (2) human PP, (3) thrombin, (4) PP + alexin, and (5) Tris buffer, shown in Figure 5A. We monitored the resulting plugs with fluorescent microscopy for $60 \mathrm{~min}$ (Figure 5B). Plug 1 did not show an increase of fluorescence intensity. Plug 2 provided the background clotting time $(15 \mathrm{~min})$ under these experimental conditions. Plugs 3 and 4 showed a significant increase in fluorescence relative to that of plug 2 within 5 min, confirming that addition of alexin shortened the clotting time. In plug 5, a very slow increase of fluorescence was detected, most likely due to cross-contamination from plug 4 . After $1 \mathrm{~h}$ of incubation, the intensity in plug 5 was 5 times lower than the average intensity of plugs 3 and 4. Intensity profiles for all five plugs over time are shown in Figure 5C. While cross-contamination tests predicted that only $0.1 \%$ of plasma and alexin should be transferred to plug 5, even a small amount of contamination could lead to an observable fluorescent signal, because thrombin is an enzyme. This result emphasizes the importance of reducing crosscontamination.

\section{CONCLUSIONS}

The multijunction device described in this paper improves upon the previously reported Tjunction by significantly reducing cross-contamination and providing a wider range of injection ratios. The physical model of transport ${ }^{1,2}$ we have used to design the multijunction device was experimentally verified and shown to predict the maximum flow rate, $Q_{\max }$, to within a factor of 2. Enzymatic reactions are especially sensitive to cross-contamination and would benefit the most from this approach. A wider range of accessible injection ratios means that this device could be applied to titrations and assays that require a wide range in volume of substrate inputs, such as protein crystallization trials. This technology is also applicable to complex biological fluids such as blood. These improvements should lead to new applications of microfluidic assays that require screening multiple conditions in nanoliter volumes. We are currently applying this technology to the crystallization of membrane proteins ${ }^{32}$ which are available only in small quantities and are challenging to handle.

\section{Supplementary Material}

Refer to Web version on PubMed Central for supplementary material.

\section{ACKNOWLEDGMENT}

This work was supported in part by the NIH Roadmap for Medical Research under Grant No. R01 GM075827-02, the NIBIB under Grant No. R01 EB001903-04, and the UC/ANL Collaborative Seed Funding. We thank Helen Song for helpful discussions and Jessica Price for assistance in writing and editing this manuscript.

\section{References}

(1). Kralj JG, Sahoo HR, Jensen KF. Lab Chip. in press

(2). Gunther A, Jhunjhunwala M, Thalmann M, Schmidt MA, Jensen KF. Langmuir 2005;21:1547-1555. [PubMed: 15697306]

(3). Kohler JM, Henkel T. Appl. Microbiol. Biotechnol 2005;69:113-125. [PubMed: 16228204] 
(4). Abkarian M, Faivre M, Stone HA. Proc. Natl. Acad. Sci. U.S.A 2006;103:538-542. [PubMed: 16407104]

(5). Janasek D, Franzke J, Manz A. Nature 2006;442:374-380. [PubMed: 16871204]

(6). Jensen K, Lee A. Lab Chip 2004;4:31N-32N.

(7). Thorsen T, Maerkl SJ, Quake SR. Science 2002;298:580-584. [PubMed: 12351675]

(8). Sia SK, Whitesides GM. Electrophoresis 2003;24:3563-3576. [PubMed: 14613181]

(9). Belder D. Angew. Chem., Int. Ed 2005;44:3521-3522.

(10). Srinivasan V, Pamula VK, Fair RB. Lab Chip 2004;4:310-315. [PubMed: 15269796]

(11). Zheng B, Tice JD, Roach LS, Ismagilov RF. Angew. Chem., Int. Ed 2004;43:2508-2511.

(12). Losey MW, Schmidt MA, Jensen KF. Ind. Eng. Chem. Res 2001;40:2555-2562.

(13). Song H, Tice JD, Ismagilov RF. Angew. Chem., Int. Ed 2003;42:768-772.

(14). Anna SL, Bontoux N, Stone HA. Appl. Phys. Lett 2003;82:364-366.

(15). Nisisako T, Torii T, Higuchi T. Lab Chip 2002;2:24-26. [PubMed: 15100856]

(16). Tice JD, Song H, Lyon AD, Ismagilov RF. Langmuir 2003;19:9127-9133.

(17). Song H, Ismagilov RF. J. Am. Chem. Soc 2003;125:14613-14619. [PubMed: 14624612]

(18). Burns JR, Ramshaw C. Lab Chip 2001;1:10-15. [PubMed: 15100883]

(19). Chan EM, Alivisatos AP, Mathies RA. J. Am. Chem. Soc 2005;127:13854-13861. [PubMed: 16201806]

(20). Nisisako T, Torii T, Higuchi T. Chem. Eng. J 2004;101:23-29.

(21). Song H, Chen DL, Ismagilov RF. Angew. Chem., Int. Ed 2006;45:7336-7356.

(22). Zheng B, Ismagilov RF. Angew. Chem., Int. Ed 2005;44:2520-2523.

(23). Xu SQ, Nie ZH, Seo M, Lewis P, Kumacheva E, Stone HA, Garstecki P, Weibel DB, Gitlin I, Whitesides GM. Angew. Chem., Int. Ed 2005;44:724-728.

(24). Hatakeyama T, Chen DLL, Ismagilov RF. J. Am. Chem. Soc 2006;128:2518-2519. [PubMed: 16492019]

(25). Gunther PM, Moller F, Henkel T, Kohler JM, Gross GA. Chem. Eng. Technol 2005;28:520-527.

(26). Shestopalov I, Tice JD, Ismagilov RF. Lab Chip 2004;4:316-321. [PubMed: 15269797]

(27). Song H, Li HW, Munson MS, Van Ha TG, Ismagilov RF. Anal. Chem 2006;78:4839-4849. [PubMed: 16841902]

(28). Roach LS, Song H, Ismagilov RF. Anal. Chem 2005;77:785-796. [PubMed: 15679345]

(29). Munson, BR.; Young, DF.; Okiishi, TH. Fundamentals of fluid mechanics. 4th ed.. John Wiley \& Sons, Inc.; New York: 2002.

(30). Eggers J, Lister JR, Stone HA. J. Fluid Mech 1999;401:293-310.

(31). Kawabata SI, Miura T, Morita T, Kato H, Fujikawa K, Iwanaga S, Takada K, Kimura T, Sakakibara S. Eur. J. Biochem 1988;172:17-25. [PubMed: 3278905]

(32). Li L, Mustafi D, Fu Q, Tereshko V, Chen DL, Tice JD, Ismagilov RF. Proc. Natl. Acad. Sci. U.S.A 2006;103:19243-19248. [PubMed: 17159147] 

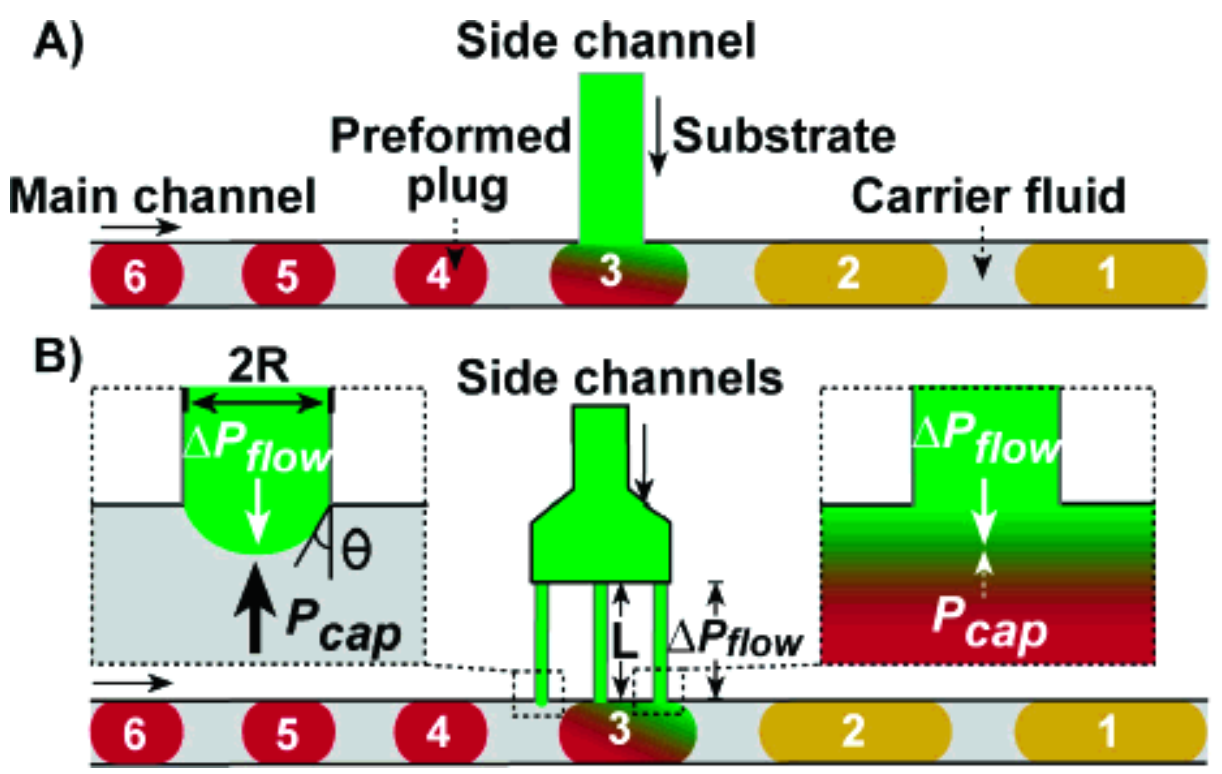

Figure 1.

Schematic drawings of two microfluidic devices used to inject a substrate into an array of preformed plugs: (A) simple T-junction device; (B) multijunction injector described in this paper. Parameters for the physical model, including $\Delta P_{\text {flow }}$ and $P_{\text {cap }}$, are shown (see the text for details). 


\section{Side channels}

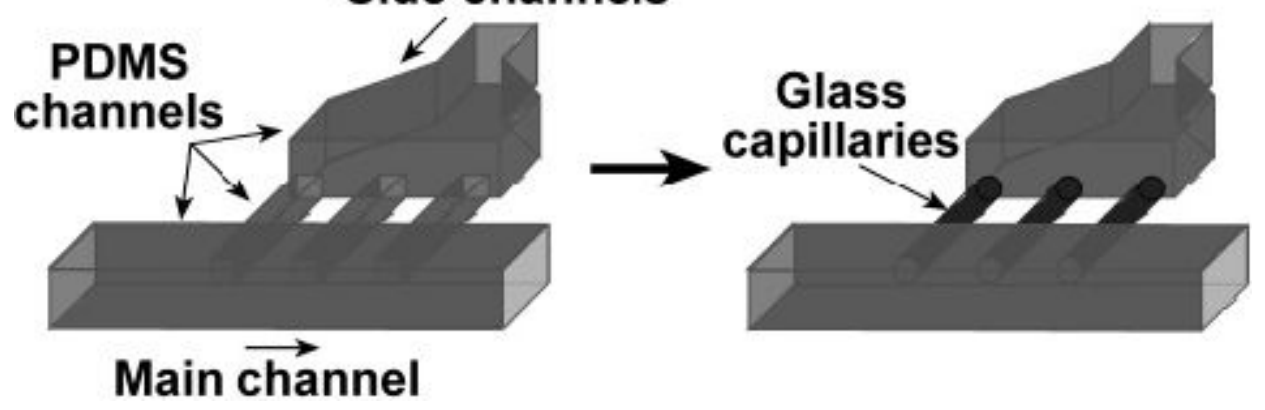

Figure 2.

Key features in the fabrication of the multijunction device. The parallel side channels of a PDMS device, made by using two-layer lithography, were replaced with glass capillaries to make the side channels hydrophilic. 


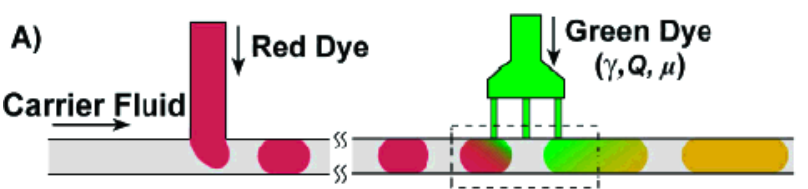

B)

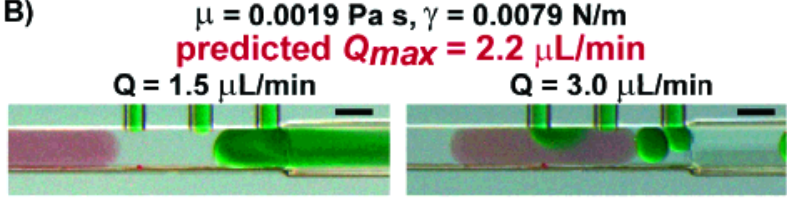

C)

$\mu=0.0019 \mathrm{~Pa} \mathrm{~s}, \gamma=0.0202 \mathrm{~N} / \mathrm{m}$ predicted $Q_{\max }=5.8 \mu \mathrm{L} / \mathrm{min}$ $Q=5.5 \mu \mathrm{L} / \mathrm{min}$
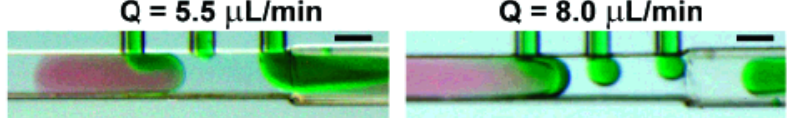

D)

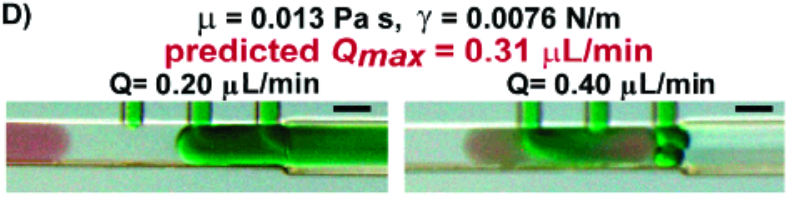

E)

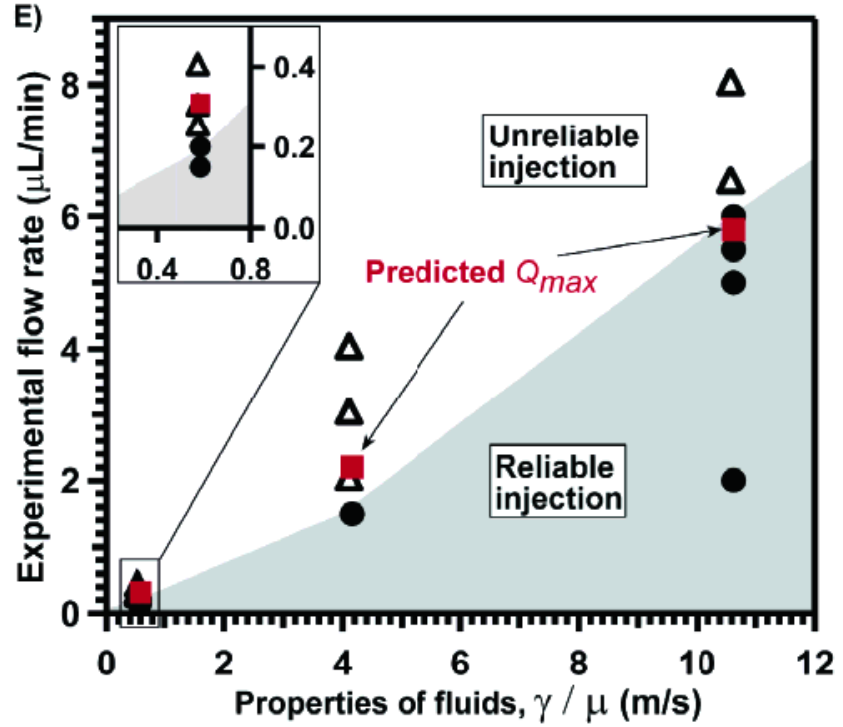

Figure 3.

Experimental validation of $Q_{\max }$ predicted by the physical model. Predicted $Q_{\max }$ values are shown in red in larger font, and experimentally observed values of $Q$ at which the transition occurs from successful injection to formation of small droplets are shown in black. (A) Schematic drawing of the experimental setup. Plugs of red dye were formed in a T-junction, and green dye was injected using the multijunction device. (B-D) Substrate solution (green dye) was injected into preformed plugs at flow rates below (left column) and above (right column) the predicted $Q_{\max }$ for various experimental conditions. Formation of small droplets of substrate solution was prevented only when the flow rate of substrate was below $Q_{\max }$ (see the text for details). (E) A diagram showing injection patterns for experiments B, C, and D. Solid circles indicate flow rates yielding reliable injection at given $\gamma / \mu$ values; open triangles indicate flow rates yielding nonreliable injection at given $\gamma / \mu$ values; red squares indicate predicted $Q_{\max }$ at given $\gamma / \mu$ values. The shaded region indicates the range of flow rates resulting in reliable injection for specific values of $\gamma / \mu$. 


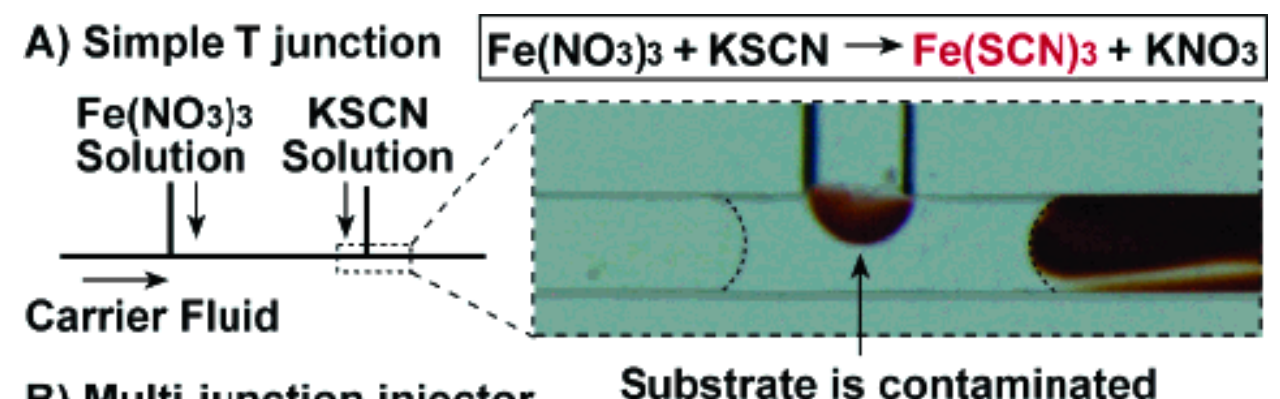

B) Multi-junction injector Substrate is contaminated

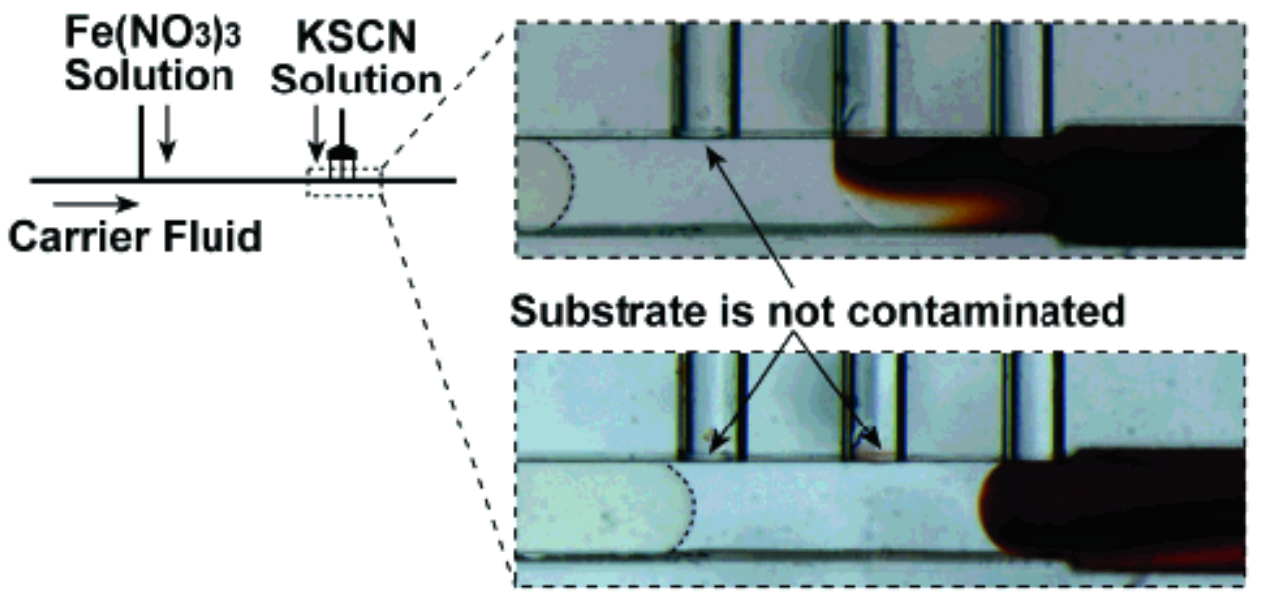

Figure 4.

Tests for cross-contamination with a color-change reaction. Injecting KSCN solution (clear) into preformed $\mathrm{Fe}\left(\mathrm{NO}_{3}\right)_{3}$ plugs (clear) resulted in red plugs $\left(\mathrm{Fe}(\mathrm{SCN})_{3}\right.$ solution). (A) The Tjunction caused cross-contamination: the red solution remained in the side channel after injection. The side channel (vertical) was a glass capillary with an i.d. of $200 \mu \mathrm{m}$. The main channel (horizontal) was a $200 \mu \mathrm{m} \times 200 \mu \mathrm{m}$ PDMS channel. (B) The multijunction device reduced cross-contamination: no red solution was observed in the side channels (glass capillaries, $100 \mu \mathrm{m}$ i.d.) after a plug passed by. The main channel was a $200 \mu \mathrm{m} \times 250 \mu \mathrm{m}$ PDMS channel that increased to $250 \mu \mathrm{m} \times 250 \mu \mathrm{m}$ after the junctions. 

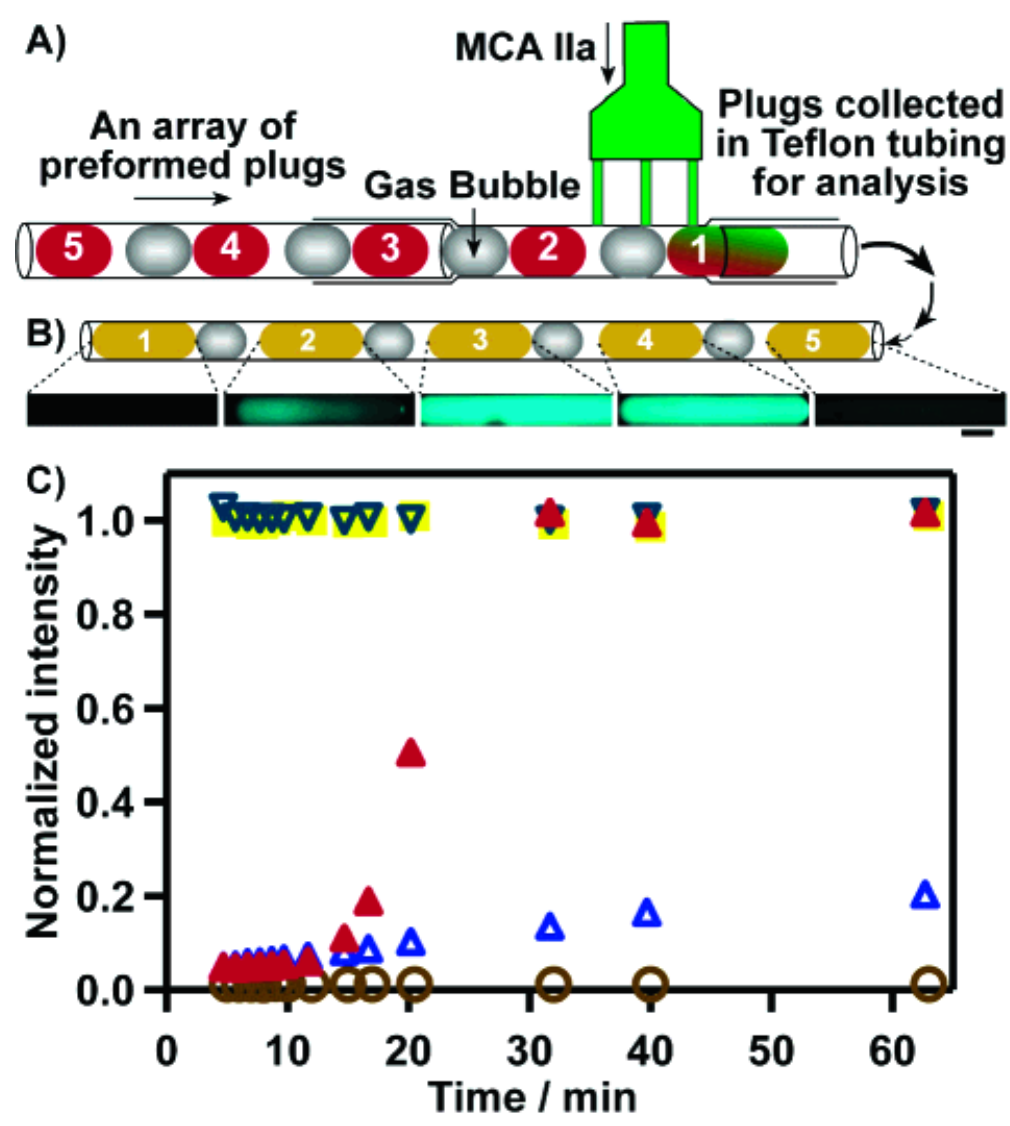

Figure 5.

A control blood assay performed with the multijunction device. (A) Schematic drawing of the experimental setup. Substrate MCA IIa was injected into five preformed plugs separated by air bubbles. The five plugs were formed in the order (1, open brown circles) Tris buffer, (2, solid red triangles) human PP, (3, solid yellow squares) thrombin, (4, open inverted triangles) $\mathrm{PP}+$ alexin, and (5, open blue triangles) Tris buffer. (B) Fluorescent images of the five resulting plugs taken at the time point of $20 \mathrm{~min}$, scale bar $200 \mu \mathrm{m}$. (C) Profiles of fluorescence intensity for five plugs in the assay. Fluorescent images were collected under the same conditions, and the fluorescence intensity was normalized the same way in all images. 